\title{
PEA SYSTEM MODELING AND SIGNAL PROCESSING FOR MEASUREMENT OF VOLUME CHARGE DISTRIBUTIONS IN THIN DIELECTRIC FILMS
}

\author{
L. H. Pearson ${ }^{(1)}$, J. R. Dennison ${ }^{(2)}$, E. W. Griffiths ${ }^{(1)}$, A. C. Pearson ${ }^{(1)}$
}

\begin{abstract}
This paper discusses our effort to develop advanced pulsed electroacoustic (PEA) measurement system capabilities that incorporate (1) improved signal processing tools for increased signal/noise ratios; and (2) integrated PEA modeling tools. In addition, we emphasize state-of-the-art system electronic components, integrated environmental controls, and sensor improvements required to achieve high spatial resolution while maintaining reasonable temporal resolution for both ambient and in vacuo measurements of thin dielectrics charged using electron beam injection, which is most applicable for spacecraft charging tests. PEA measurement systems provide an important tool to investigate the spatial extent and dynamic evolution of embedded charge distributions in thin dielectric materials. This knowledge has important applications in spacecraft industries, as well as for semiconductors, high-power electronic devices, high-voltage DC power cable insulation, and high-energy and plasma physics apparatus. The emphasis of this paper is on improved signal processing methods and integrated PEA modeling tools.
\end{abstract}

Index Terms-Pulsed electro acoustics, ultrasonics, signal processing.

\section{APPROACH}

Pulsed Electro-Acoustic (PEA) measurement techniques are nondestructive and, arguably, one of the most promising methods to provide the desired information on internal charge distributions related to spacecraft charging issues [1,2]. PEA techniques generate acoustic waves within the material by using high voltage, high frequency, pulsed signals to stimulate motion of the internal charge bound in the solid. External piezoelectric transducers convert the acoustic signal generated in this manner to an electrical signal for detection and analysis. Signal processing methods are applied to improve signal-to-noise ratio (SNR). Further, modeling tools are needed to interpret measured waveforms, to guide experimental design, and to support trouble-shooting efforts.

\section{IMPROVED SIGNAL PROCESSING}

With most raw waveform measurements, some level of

This work was supported by Small Business Technology Transfer Research (STTR) Phase I and Phase II funding from the Air Force Research Laboratory, Kirtland Air Force Base, Albuquerque, New Mexico, USA.

Lee Pearson, Erick Griffiths and Anthony Pearson are with Box Elder Innovations, LLC, in Bear River City, UT, 84301 USA, (email: beinnov@frontier.com ).

JR Dennison is with the Materials Physics Group in the Physics Department at Utah State University in Logan, UT 84322 USA (email: JR.Dennison@usu.edu).

Color versions of one or more figures in this paper are available online at http://ieeexplore.ieee.org.

Digital object identifier. signal processing has been found to be necessary in order to improve the SNR to a satisfactory level. Signal processing methods developed in this program are described below, including: averaging, band-pass filter, split spectrum processing, and deconvolution. These methods are not only helpful in reducing noise, but also in improving spatial resolution.

\section{$2.1 \quad$ Averaging}

The most common method for improving the SNR is accomplished by simply averaging waveforms. Improvement in the SNR from averaging is proportional to the square root of the number of waveforms averaged. Hence, there is a practical limit to the use of averaging as a viable signal processing tool because of the time commitment required and the diminishing returns from averaging greater and greater numbers of waveforms. Additional signal processing tools can be applied to add further improvement to the integrity of measured waveforms. Some of these are described below.

\subsection{Band-Pass Filtering}

This method applies a frequency domain Gaussian filter to the data using forward and inverse fast Fourier transforms. The frequency domain response is multiplied by a Gaussian filter which is characterized by its center frequency $\left(f_{0}\right)$ and full width at half amplitude or band width $(B W)$. The Gaussian filter is modified by a sine function to ensure that it goes to zero as the frequency $(f)$ goes to zero. The filter is given by:

$$
\operatorname{Filter}(f)=e^{-\frac{4 \ln (2)}{B W^{2}}\left(f-f_{0}\right)^{2}} \sin \left(\frac{\pi}{2 f_{0}} f\right)
$$

\subsection{Split Spectrum Processing (SSP)}

This method was developed to reduce noise and clutter in ultrasonic waveforms resulting from grain scatter in nondestructive testing of metals or other heterogeneous materials with microstructure that causes scattering [3]. SSP also works well to suppress random electronic noise in ultrasonic signals. The method contains the following steps:

(i) The raw waveform is Fourier transformed into frequency-domain and split into $\mathrm{N}$ wavelets using overlapping Gaussian band-pass filters.

(ii) Wavelets are transformed back into timedomain.

(iii) At each time step or element in the wavelets, the average value and standard deviation are calculated from which the coefficient of variation ( $\mathrm{CV}=$ stdev/avg) is obtained.

(iv) The original waveform is then divided at each time element by the corresponding CV. 


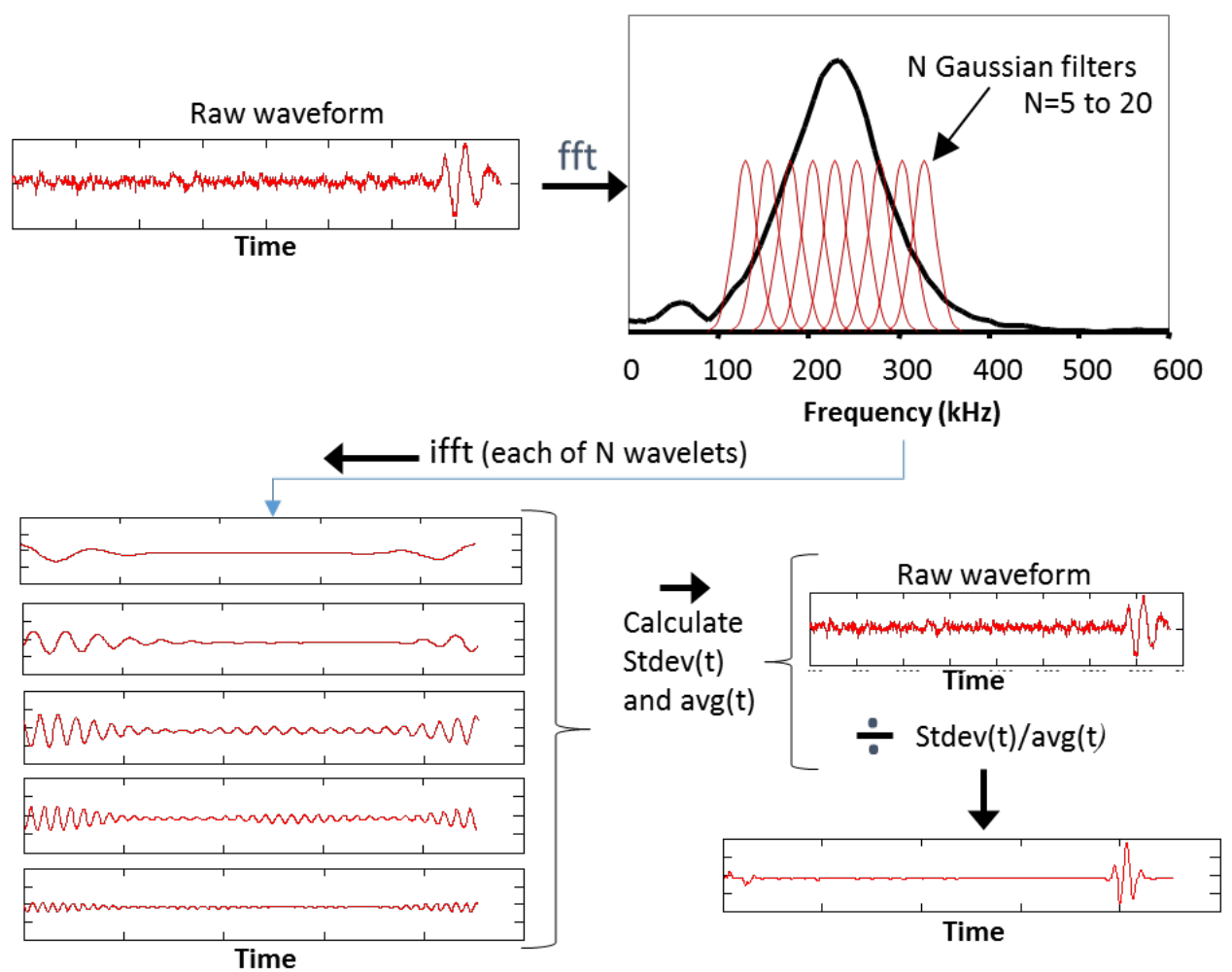

Fig. 1. Graphical representation of split spectrum processing method.

The result is that in regions where there is a coherent signal, the CV is small and the signal is increased. In regions where noise dominates, the $\mathrm{CV}$ is large and the signal is reduced. Fig. 1 graphically shows this process.

\subsection{Deconvolution}

This method is not only for improving SNR, but also for eliminating the effects of the instrumentation/sensor response function from the waveform. Deconvolution amounts to dividing out this response in frequencydomain. The measured waveform is a convolution of the instrument response function, $h(t)$, the material response function (what we want), $x(t)$, plus noise, $n(t)$, expressed by:

$$
y(t)=h(t) * x(t)+n(t)
$$

In frequency-domain, this expression is written:

$$
Y(\omega)=H(\omega) X(\omega)+N(\omega) \quad,
$$

where each function is the Fourier transform of its timedomain counterpart. A workable solution to Eq. 3 is given by the Wiener deconvolution [4] which is mathematically written,

$$
X(\omega)=\frac{Y(\omega) H^{*}(\omega)}{|H(\omega)|^{2}+Q^{2}|H(\omega)|_{\max }^{2}},
$$

where the expression $Q^{2}|H(\omega)|_{\max }^{2}$ is a measure of the noise. $Q$ is an adjustable parameter. Deconvolution is the final step in converting the waveform into a signal that has the shape of the charge distribution.
In summary, Fig. 2 shows the signal processing and data analysis process going from a raw waveform to a charge distribution and also shows electric and potential field calculations. The waveform is from a $125 \mu \mathrm{m}$ thick polyimide (Kapton $\mathrm{HN}^{\mathrm{TM}}$ ) sample with no embedded charge, with $8 \mathrm{kV}$ DC applied across the electrodes, and a 0.5 nsec width excitation pulse.

\section{PEA WAVEFORM MODELING}

PEA waveform modeling is helpful in determining the effects and relative importance of the physical parameters that are inputs to the model (e.g., acoustic properties, material thicknesses, density, etc.) and hence in a PEA waveform measurement. It also gives insight into modifications to the PEA method that can be used to improve or better understand the method. Two models are presented: i) a ray-tracing model similar to many published models [1,5-15], and ii) a full-wave model based on computing fields by satisfying boundary conditions at each interface [16,17].

\subsection{Ray-Tracing Model}

In this model, dominant and first arriving wavelets are individually traced through the sample and adjacent material layers in the sample holding fixture and then mathematically summed, using the principle of linear superposition, to give a complete waveform that is equivalent to the measured waveform. Most literature models only account for the initial wavelet generated by the charge layer embedded in the dielectric film. However, there are many additional wavelets coming from the sample that contain information about the charge distribution and the acoustic properties of the material layers. Fig. 3 shows a conceptual diagram of a generic 

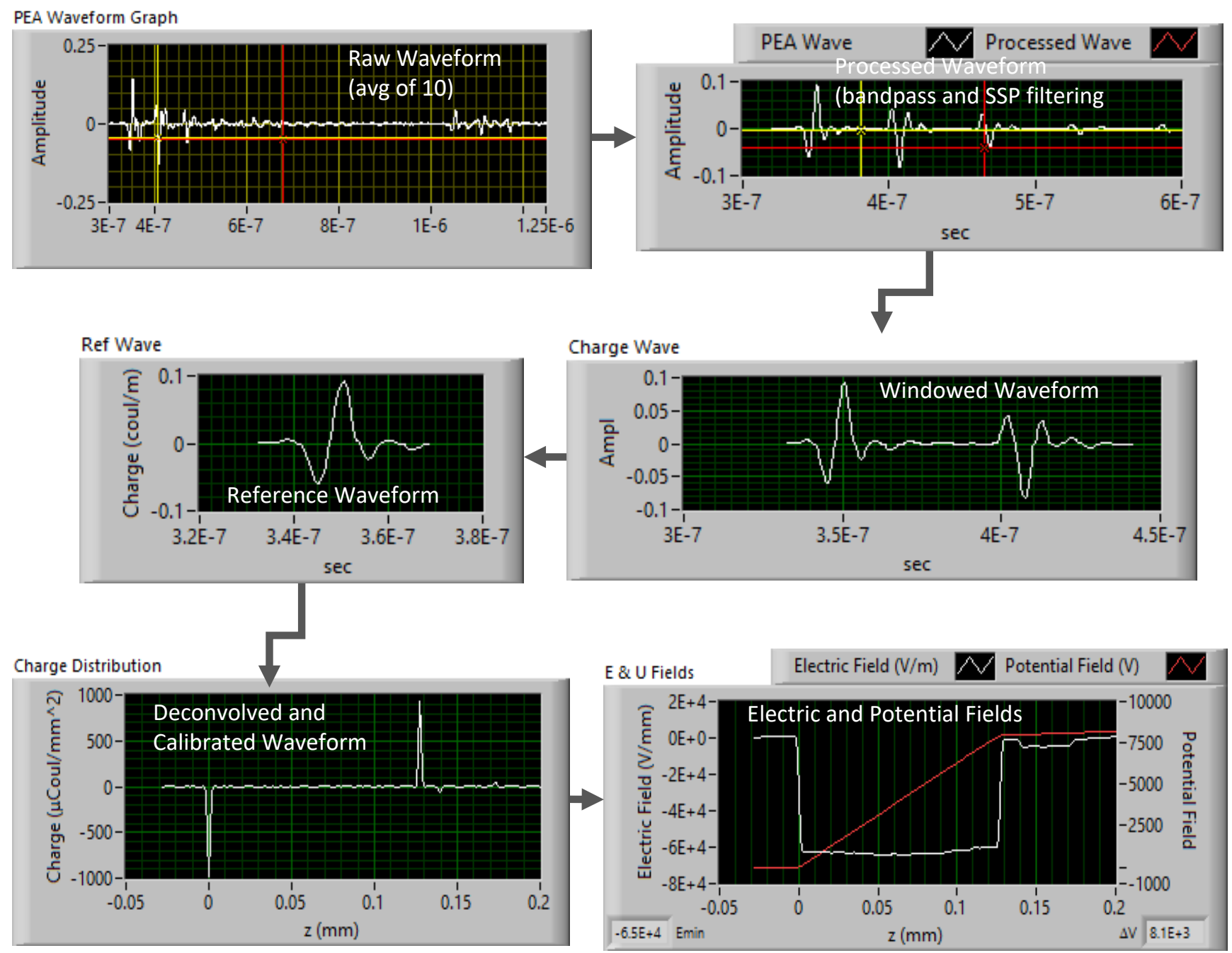

Fig. 2. Flow diagram showing signal processing and data analysis process

PEA test setup including (not to scale) the layered material system made up of the charged dielectric film, aluminum electrodes, and piezoelectric sensor.

A brief outline of the theoretical basis of the model is presented below. The mathematical development process for the primary ray generated from a simulated embedded charge layer is outlined and includes the following key elements:

(i) The piezoelectric sensor is used to detect the wave generated by the electric field impulse, $\mathrm{E}(\mathrm{t})$.

(ii) A negative charge layer (distribution) is embedded in the dielectric film (layer) and for simplicity, is assumed to have a shape approximated by a Maxwellian distribution function.

(iii) Charge layers are induced on the conducting electrodes on each side of the dielectric layer.

(iv) Two acoustic waves are generated by each charge layer from the force caused by $E(t)$ and propagate in opposite directions from the charge layer and with opposite phase.

Constraining assumptions in the mathematical development include: (i) The thickness of each layer is small compared to its lateral dimensions.

(ii) The lateral dimensions are large compared to the wavelength of the induced ultrasonic wave.

(iii) Assumptions (i) and (ii) lead to the assumption that there are no guided wave modes and that the problem can be treated as one-dimensional.

(iv) All acoustic waves are bulk longitudinal waves propagating normal to the plane of each layer.

(v) The charge distribution in the dielectric film only has a $z$-dependence (normal to the sample plane).

(vi) The aluminum electrode between the sensor and the dielectric layer is thick enough so that all multiple reflections within that layer are far enough out in time that they can be ignored.

The volume charge distribution in the dielectric layer is given by, $\rho(\mathrm{z})$. A force acts on the charge layer when an external pulsed electric field, $E(t)$, is applied. The force on a thin sub-layer of charge of thickness, $\Delta z$, at location $\mathrm{z}$ relative to the left side of the dielectric film, is given by:

$\Delta f(z, t)=\rho(z) \cdot \Delta z \cdot E(t) \quad$

In the frequency domain, this expression can be written: 


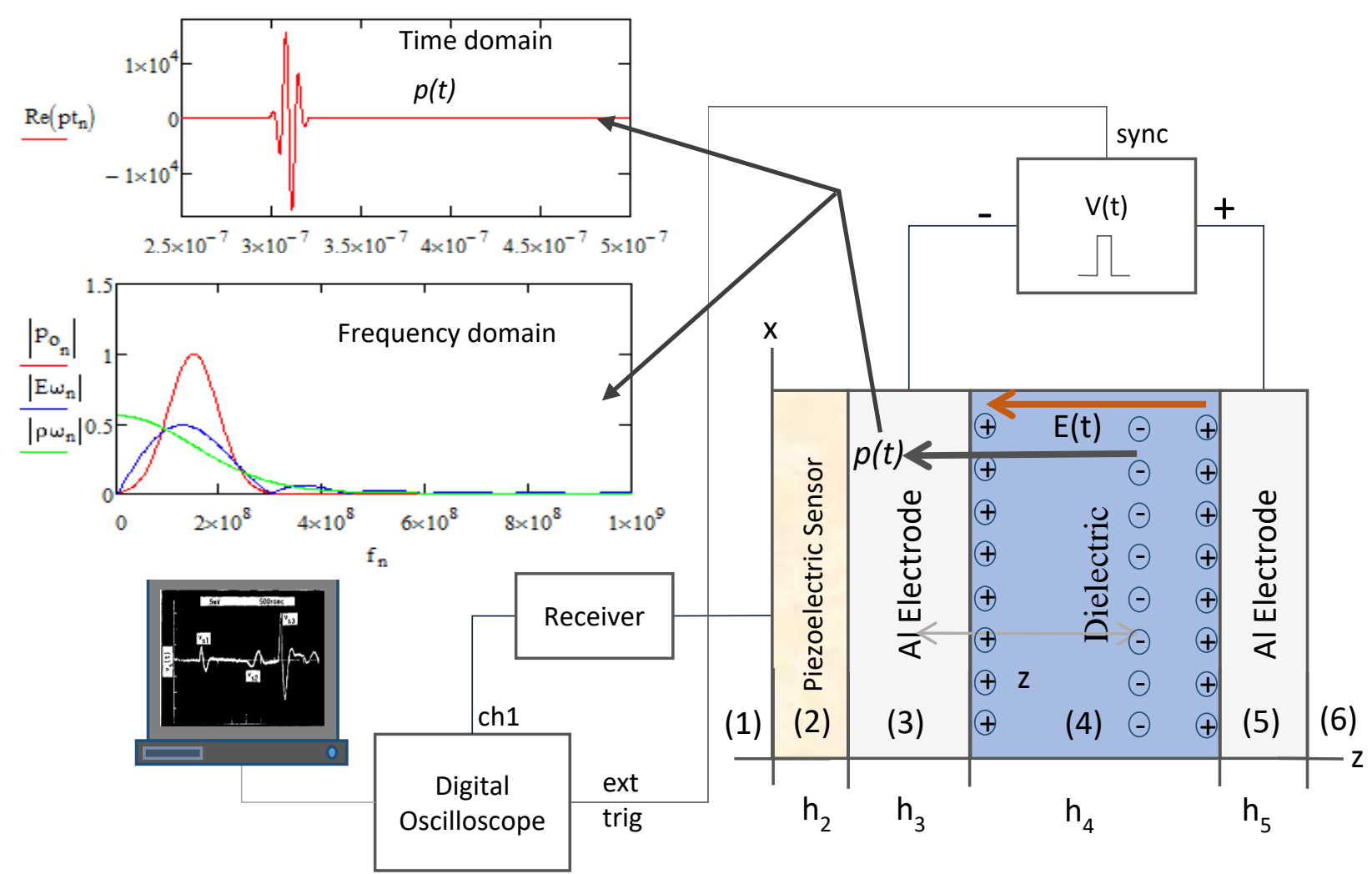

Fig. 3. Conceptual diagram showing a generic PEA setup with a charged dielectric film, electrodes, sensor, and basic equipment components.

$\Delta f(\omega, z)=\rho(z) \cdot \Delta z \cdot E(\omega)$

where $\Delta f(\omega, z)$ and $E(\omega)$ are Fourier transforms of $\Delta f(z, t)$ and $E(t)$, respectively. The force (over the cross sectional area) creates a pressure wave that propagates to the piezoelectric sensor and is given by:

$\Delta p(\omega, z)=p_{0}(\omega) \cdot \rho(z) \cdot \Delta z \cdot E(\omega) \cdot e^{i k_{4} z} \cdot e^{i k_{3} h_{3}} \cdot t_{43} \cdot t_{32}$

where $p_{0}(\omega)$ is the transducer/instrumentation response function and is assumed, for convenience, to have a Gaussian response shape in frequency domain. $t_{43}$ and $t_{32}$ are the transmission coefficients for the dielectric/electrode and electrode/piezoelectric sensor interfaces, respectively (refer to Fig. 3). The exponential factors are added to account for the phase shifts (or time delays in time domain) for each respective layer. The $z$ coordinate can be transformed to a time coordinate with the following substitutions:

$z=t \cdot c_{4} ; \Delta z=c_{4} \Delta t ; k_{4}=\frac{\omega}{c_{4}}$,

where $c_{4}$ is the wavespeed in the dielectric, $\omega$ is the angular frequency, and $k_{4}$ is the wavevector in the dielectric. Eq. 3 can now be written as

$$
\Delta p(\omega, t)=p_{0}(\omega) \cdot E(\omega) \cdot c_{4} \cdot e^{i k_{3} h_{3}} \cdot t_{43} \cdot t_{32} \cdot \rho\left(c_{4} t\right) \cdot e^{i \omega t} \Delta t
$$

Summing up all the wavelets from each charge sub-layer $(\Delta z)$ is accomplished by integrating as follows:

$$
p(\omega)=p_{0}(\omega) \cdot E(\omega) \cdot c_{4} \cdot e^{i k_{3} h_{3}} \cdot t_{43} \cdot t_{32} \cdot \int_{0}^{t} \rho\left(c_{4} t\right) \cdot e^{i \omega t} \cdot d t
$$

The limits on the integral can be extended to $\pm \infty$ without changing the results of the integration, because the charge is contained within the dielectric layer. The integral now becomes a Fourier integral and Eq. 10 can now be written in terms of the Fourier transform of the charge distribution, $\rho(\omega)$ :

$p(\omega)=p_{0}(\omega) \cdot E(\omega) \cdot c_{4} \cdot e^{i k_{3} h_{3}} \cdot t_{43} \cdot t_{32} \cdot \rho(\omega)$

Eq. 11 is the stress transferred to the sensor from the charge layer. The stress, $\sigma q$, from the embedded charge in the dielectric layer is then given by

$p(\omega)=E(\omega) \cdot c_{4} \cdot \rho(\omega)=\sigma q$

Mathematically, the time delay (phase shift) due to the zdependence of the charge distribution integrates to $\rho(\omega)$, so a complex phase factor (exponential) is no longer explicit in the expression for the wave packet, $p(\omega)$. The time-domain waveform (shown in Fig. 3) is obtained by performing an inverse Fourier transform on $p(\omega)$ from Eq. 12 and then taking the real-part:

$p(t)=\operatorname{Re}[i c f f t[p(\omega)]]$ 
(1)

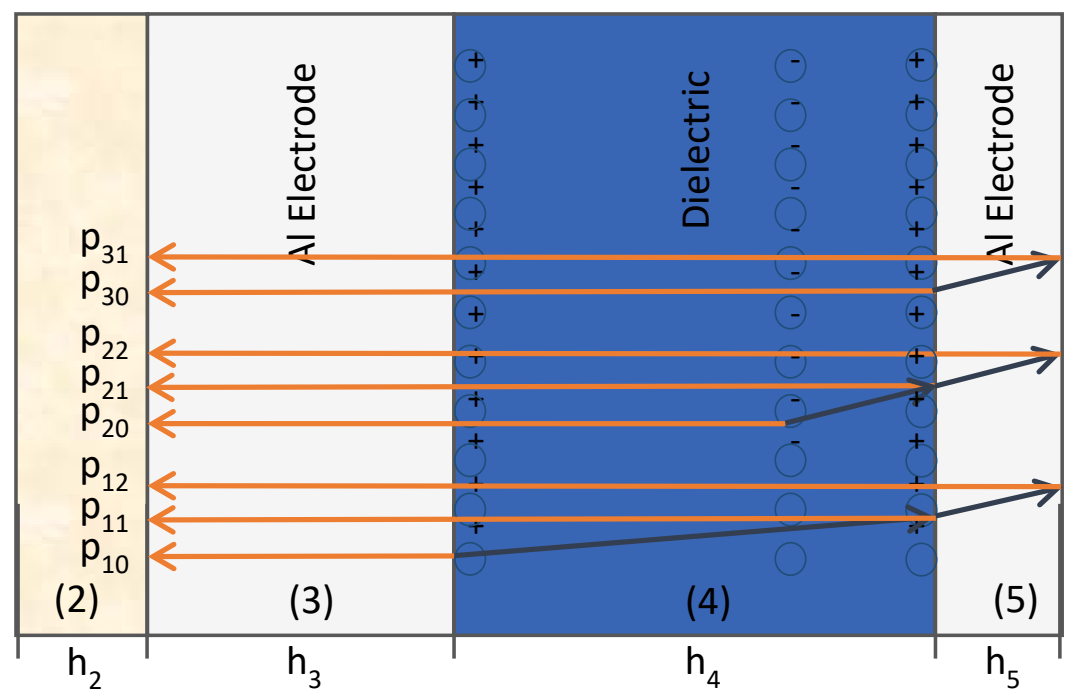

Fig.4. Drawing showing additional wavelets including additional reflecting wavelets and wavelets coming from induced charge layer.

where icfft represents an inverse, complex, fast Fourier transform. Eq. 13 represents the forward calculation model and contains only the primary wavelet created from the charge layer, which transmits directly from the embedded charge layer to the sensor and does not contain any multiple reflections/transmissions. A calculated waveform is shown in Fig. 3. To obtain the charge distribution, Eq. 11 is solved for $\rho(\omega)$ yielding

$$
\rho(\omega)=\frac{p(\omega) \cdot e^{-i k_{3} h_{3}}}{p_{0}(\omega) \cdot E(\omega) \cdot c_{4} \cdot t_{43} \cdot t_{32}}
$$

This result is inverse Fourier transformed to obtain $\rho(t)$. Eq. 14 represents a deconvolution process for obtaining $\rho(\omega)$ and this result is transformed to $\rho(\mathrm{z})$ by using Eq. 8 . The calculated frequency domain components, $p_{0}(\omega)$, $E(\omega)$, and $\rho(\omega)$ are also shown in Fig. 3. $p_{0}(\omega)$ is calculated assuming a Gaussian frequency response function for the electronic components, $E(\omega)$ is calculated assuming a one-cycle tone burst, and $\rho(\omega)$ is computed from Eq. 14.

Actual measured PEA waveforms show additional waves existing in the total "wave train" that come from the sample and other layers. These originate from multiple reflections and transmissions, and waves coming from the induced charge layers at the electrode/dielectric film interfaces. Graphically, some of these other waves are shown in Fig. 4. Assuming the principle of linear superposition, the total waveform can be obtained by summing all potential waves. Each wave must have an explicit mathematical expression in order to complete the sum. The derivation leading to Eqs. 11-13 is for the wave designated by $p_{20}$ in Fig. 4 . The wavelets $p_{10}$ and $p_{30}$, for the waves generated from the induced charges on the grounded and positive electrodes, respectively, for example, can be written as:

$$
p_{10}(\omega)=p_{0}(\omega) \cdot E(\omega) \cdot e^{i k_{3} h_{3}} \cdot t_{32}
$$

$p_{30}(\omega)=p_{0}(\omega) \cdot E(\omega) \cdot c_{4} \cdot \rho\left(c_{4} \omega\right) \cdot e^{i k_{3} h_{3}} \cdot t_{43} \cdot t_{32}$

Additional wavelets, shown in Fig. 4, can be similarly written where wavelets with numbering formats $p_{1 x}$ or $p_{3 x}$ are for wavelets originating at the grounded and positive electrodes, respectively, and wavelets with the numbering format $p_{2 x}$ originate from the embedded charge layer. Each wavelet takes a different path through the layered media, however, all wavelets are traveling normal to the plane of each layer. In theory, there are an infinite number of these addition wavelets that can be traced through the layered media, but in practice, only a small number of them are needed to represent the measured waveform because the remaining wavelets are at the noise level or are too far out in time to be of interest.

\subsection{Full-Wave Model}

As stated, the theory developed in the previous section is a ray-tracing approach to deriving PEA equations for predicting waveforms. A more general approach, or "full-wave model" is developed below using mathematics modified from that described in reference 17 for a similar application of having an embedded piezoelectric layer in a layered medium. The advantage of this full-wave model approach is that all rays are implicitly accounted for in the theory. This model is presented below. The full-wave model and portions of the ray-tracing have been implemented in a LabVIEW PEA control program developed in this effort. The theoretical development goes as follows:

For the thickness-mode sensor used in the PEA application, the voltage (assumed to be sinusoidal) across the electrodes of the sensor is given by

$V(\omega)=\int_{0}^{h} E(\omega, z) d z$

where 


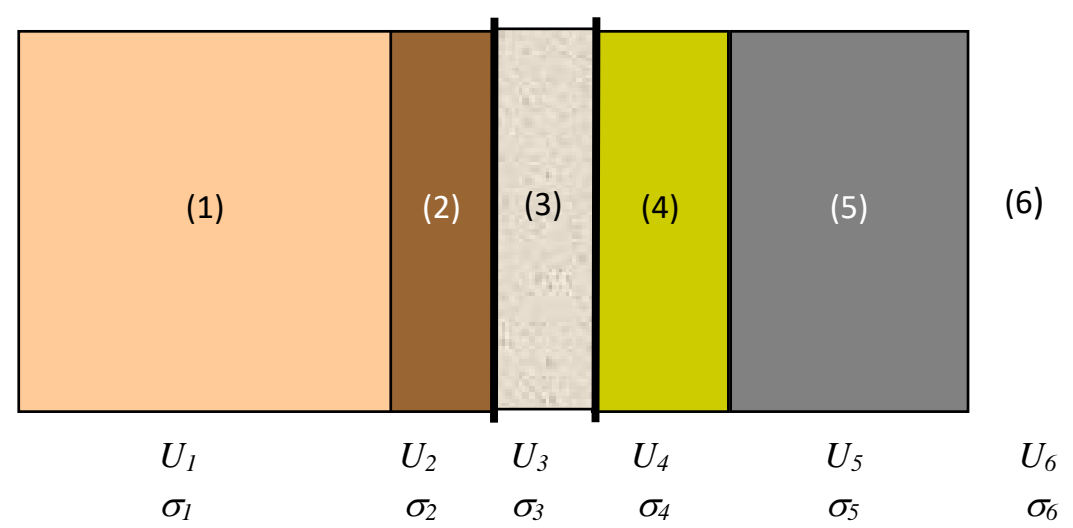

Fig. 5. Diagram showing N-Layered media, corresponding displacement, and stress.

$E(\omega, z)=-h_{33} \frac{d}{d z} U(\omega, z)+\beta_{33} D(\omega, z)$

and where

$E=$ z-component of electric field

$D=z$-component of the electric induction $(D=\varepsilon E)$

$U=z$-component of the displacement (relates to change in thickness)

$h=$ thickness of piezoelectric sensor

$\beta_{33}=$ z-component of the dielectric impermeability tensor (inverse of permeability tensor).

The normal component of the electric induction field is

$D_{Z}=\vec{D} \cdot \hat{n}=\sigma_{C}$

where $\sigma_{C}$ is the surface charge density. The time dependence of $D_{Z}$ is given by

$D_{Z}=D_{0} e^{i \omega t}$.

Taking a derivative of $D_{Z}$ with respect to time gives the following:

$\frac{d}{d t} D_{Z}=i \omega D_{Z}=\frac{d}{d t} \sigma_{C}=\frac{I(\omega)}{A_{0}}$

where

$\omega=$ angular frequency $=2 \pi f$

$I(\omega)=$ electric current

$A_{O}=$ surface area of piezoelectric sensor

Thus,

$D_{Z}=\frac{I(\omega)}{i \omega A_{0}}$

Substituting (21) into (18) and then (18) into (17), then integrating gives:

$V(\omega)=h_{33}(U(\omega, 0)-U(\omega, h))+\frac{\beta_{33} I(\omega) h}{i \omega A_{0}}$

The displacement, $U$, is found by taking the general solution to the wave equation and satisfying boundary conditions listed below. The wave equation is:

$\frac{d^{2}}{d z^{2}} U(\omega, z)-\frac{\rho \omega^{2}}{C_{33}} U(\omega, z)=0$

The general solution has the form:

$U(\omega, z)=A e^{i k z}+B e^{-i k z}$

where $A$ and $B$ are constants to be determined by matching solutions across boundaries using the boundary conditions and $k$ is the wavevector given by

$k^{2}=\frac{\rho \omega^{2}}{C_{33}} \quad$ or $\quad C_{33}=\rho \frac{\omega^{2}}{k^{2}}$

where $k=\omega / c+i \alpha, c$ is the wave speed, and the attenuation coefficient $\alpha=$ constant $\cdot \omega$.

The assumption is made that the wave speed is constant with respect to frequency and that the attenuation coefficient is proportional to frequency. The boundary conditions for the case of longitudinal waves are: (1) normal components of stress are continuous, and (2) normal components of displacement are continuous. For piezoelectric layers, the normal stress is given by $\sigma_{Z}=C_{33} \frac{d}{d z} U(\omega, z)-h_{33} D_{Z}$

and for layers with embedded charge, the normal stress is given by (see (12))

$\sigma_{Z}=C_{33} \frac{d}{d z} U(\omega, z)+\sigma_{q}$

Ohm's law is given by,

$Z(\omega)=\frac{V(\omega)}{I(\omega)}$

where $Z(\omega)$ is the complex electrical impedance. For $N$ layers, there are $N-1$ boundaries or interfaces (see Fig. 5) and, hence, $2 \cdot(N-1)$ boundary conditions as listed below: 


\begin{tabular}{|c|c|}
\hline$U_{1}=U_{2}$ & $z=0$ \\
\hline$\sigma_{\mathrm{z} 1}=\sigma_{\mathrm{z} 2}$ & $z=0$ \\
\hline$U_{2}=U_{3}$ & $z=h_{2}$ \\
\hline$\sigma_{z 2}=\sigma_{z 3}$ & $z=h_{2}$ \\
\hline$\cdot$ & \\
\hline$\dot{\cdot}$ & \\
\hline$U_{j}=U_{j+1}$ & \\
\hline$\sigma_{z j}=\sigma_{z j+1}$ & \\
\hline$\cdot$ & \\
\hline 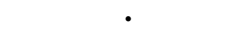 & \\
\hline$U_{N-2}=U_{N-1}$ & $z=h_{N-2}$ \\
\hline$\sigma_{Z N-2}=\sigma_{Z N-1}$ & $z=h_{N-2}$ \\
\hline$U_{N-1}=U_{N}$ & $z=h_{N-1}$ \\
\hline$\sigma_{Z N-1}=\sigma_{Z N}$ & $z=h_{N-1}$ \\
\hline
\end{tabular}

where $h_{j}$ is the thickness of layer $j$. Applying the boundary conditions leads to the following results:

(i) At $z=h_{1}\left(h_{1}=0, A_{1}=0\right.$ because there is no returning wave from the far left):

$$
\begin{aligned}
& A_{1} e^{-i k_{1} h_{1}}=A_{2}+B_{2} \\
& -i k_{1} C 33_{1} B_{1} e^{-i k_{1} h_{1}}-h 33_{1} D z_{1}+\sigma q_{12} \\
& =i k_{2} C 33_{2} A_{2}-i k_{2} C 33_{2} B_{2}-h 33_{2} D z_{2}+\sigma q_{2}
\end{aligned}
$$

(ii) At $z=h_{2}$ :

$$
\begin{aligned}
& A_{2} e^{i k_{2} h_{2}}+B_{2} e^{-i k_{2} h_{2}}=A_{3}+B_{3} \\
& -i k_{2} C 33_{2} A_{2} e^{-i k_{2} h_{2}}-i k_{2} C 33_{2} B_{2} e^{-i k_{2} h_{2}}-h 33_{2} D z_{2}+\sigma q_{2} \\
& =i k_{3} C 333_{3} A_{3}-i k_{3} C 33_{3} B_{3}-h 333_{3} D z_{3}+\sigma q_{3} \\
& \text { (iii) At } z=h_{j}: \\
& A_{j} e^{i k_{j} h_{j}}+B_{j} e^{-i k_{j} h_{j}}=A_{j+1}+B_{j+1} \\
& i k_{j} C 33_{j} A_{j} e^{i k_{j} h_{j}}-i k_{j} C 33_{j} B_{j} e^{-i k_{j} h_{j}}-h 33_{j} D z_{j}+\sigma q_{j} \\
& =i k_{j+1} C 33_{j+1} A_{j+1}-i k_{j+1} C 33_{j+1} B_{j+1}-h 33_{j+1} D z_{j+1}+\sigma q_{j+1}
\end{aligned}
$$

(iv) At $\mathrm{z}=\mathrm{h}_{\mathrm{N}-2}$ :

$$
\begin{aligned}
& A_{N-2} e^{i k_{N-2} h_{N-2}}+B_{N-2} e^{-i k_{N-2} h_{N-2}}=A_{N-1}+B_{N-1} \\
& i k_{N-2} C 33_{N-2} A_{N-2} e^{i k_{N-2} h_{N-2}}-i k_{N-2} C 33_{N-2} B_{N-2} e^{-i k_{N-2} h_{N-2}} \\
& \quad-h 33_{N-2} D z_{N-2}+\sigma q_{N-2} \\
& =i k_{N-1} C 33_{N-1} A_{N-1}-i k_{N-1} C 33_{N-1} B_{N-1}-h 33_{N-1} D z_{N-1}+\sigma q_{N-1}
\end{aligned}
$$

(v) At $z=h_{N-1}\left(B_{N}=0\right.$, because there is no returning wave from the far right):

$$
\begin{aligned}
& A_{N-1} e^{i k_{N-1} h_{N-1}}+B_{N-1} e^{-i k_{N-1} h_{N-1}=A_{N}} \\
& i k_{N-1} C 33_{N-1} A_{N-1} e^{i k_{N-1} h_{N-1}}-i k_{N-1} C 33_{N-1} B_{N-1} e^{-i k_{N-1} h_{N-1}} N-1 \\
& \quad-h 33_{N-1} D z_{N-1}+\sigma q_{N-1} \\
& \quad=i k_{N} C 33{ }_{N} A_{N}-h 33_{N} D z_{N}+\sigma q_{N}
\end{aligned}
$$

If layer $j$ is the piezoelectric sensor material, (29) can be put in the following matrix format:

$Q \cdot X=(h 33 \cdot D z) Y$

where $Q$ is a square matrix of dimension $2 \cdot(\mathrm{N}-1) \times 2 \cdot(\mathrm{N}$ 1 ), and $X$ and $Y$ are vectors of length $2 \cdot(N-1)$ and are given by the following:

(i) Interface 1 [rows $n=1$ and 2]:

$$
\begin{aligned}
& Q_{1,1}=e^{-i k_{\mathbf{1}} h_{1}} \\
& Q_{1,2}=-1 \\
& Q_{1,3}=-1 \\
& Q_{2,1}=-i k_{1} C 33_{1} e^{-i k_{1} h_{1}} \\
& Q_{2,2}=-i k_{2} C 33_{2} \\
& Q_{2,3}=i k_{2} C 33_{2} \\
& Q_{1, m}=Q_{2, m}=0 \\
& 4 \leq m \leq 2(N-1)
\end{aligned}
$$

(ii) Interfaces $L=2$ to $N-2$ [rows $n=3$ to $2 \cdot(N-2)$, columns $m=2 \cdot(L-1) \ldots 2 \cdot(L-1)+3]$ :

$$
\begin{aligned}
& Q_{n, m}=e^{i k_{L} h_{L}} \\
& Q_{n, m+1}=e^{-i k_{L} h_{L}} \\
& Q_{1, m+2}=-1 \\
& Q_{1,3}=-1 \\
& Q_{n+1, m}=i k_{L} C 33_{L} e^{i k_{L} h_{L}} \\
& Q_{n+1, m+1}=-i k_{L} C 33_{L} e^{-i k_{L} h_{L}} \\
& Q_{n+1, m+2}=-i k_{L+1} C 33_{L+1} \\
& Q_{n+1, m+3}=i k_{L+1} C 33_{L+1} \\
& Q_{n, m}=Q_{n+1, m}=0 \\
& 2(L-1) \geq m \geq 2(L-1)+3
\end{aligned}
$$

(iii) Layer $L=N-1$ [rows $n=2 \cdot(N-1)-1$ to $2 \cdot(N-1)$, columns $m=2 \cdot(L-1) \ldots 2 \cdot(L-1)+2]$ :

$$
\begin{aligned}
& Q_{n, m+2}=-1 \\
& Q_{n, m}=e^{i k_{N-1} h_{N-1}} \\
& Q_{n, m+1}=e^{-i k_{N-1} h_{N-1}} \\
& Q_{n+1, m}=-i k_{N-1} C 33_{N-1} e^{-i k_{N-1} h_{N-1}}
\end{aligned}
$$




$$
\begin{aligned}
& Q_{n+1, m+1}=-i k_{N-1} C 33_{N-1} e^{-i k_{N-1} h_{N-1}} \\
& Q_{n+1, m+2}=i k_{N} C 33_{N} \\
& Q_{n, m}=Q_{n+m}=0 \\
& m \leq 2(N-1)
\end{aligned}
$$

The $\mathrm{X}$ matrix is a column matrix made up of the coefficients: $B_{1}, A_{2}, B_{2}, \ldots, A_{N-1}, B_{N-1}, A_{N}$. The displacement field $(\mathrm{Dz})$ is zero in all layers except the piezoelectric material layer, jp, and the dielectric layer with the embedded charge distribution is assumed to be in layer $j c$. With this, the matrix $Y$ is given by:

$$
\begin{aligned}
& Y_{2(j p-1)}=-1 \\
& Y_{m}=0 \quad m \neq 2(j p-1), 2 j p \\
& Y_{2 j p}=1 \\
& Y_{2 j c}=-\sigma q_{j c} \\
& Y_{2(j c-1)-2}=\sigma q_{j c-1} \\
& Y_{2 j c-2}=-\sigma q_{j c-1}+\sigma q_{j c}
\end{aligned}
$$

To complete the derivation, $\sigma q_{j c}$ and $\sigma q_{j c-1}$ are initially set to zero. This leads to a working solution. Without this assumption, an explicit, analytical solution cannot be obtained.

To get the net displacement across the piezoelectric sensor, the constants $A_{j p}$ and $B_{j p}$ are needed. They are obtained from:

$$
X=Q^{-1} Y
$$

Thus (using (21)),

$$
\begin{aligned}
& A_{j p}=h 33 \cdot D z \cdot X_{2(j p-1)}=-\frac{h 33 \cdot I(\omega)}{i \omega A_{0}} X_{2(j p-1)} \\
& B_{j p}=h 33 \cdot D z \cdot X_{2}(j p-1)+1=-\frac{h 33 \cdot I(\omega)}{i \omega A_{0}} X_{2(j p-1)+1} .
\end{aligned}
$$

Substituting (36) into (24) and evaluating at $z=0$ and $z=h_{j}$ gives:

$$
\begin{aligned}
& U(\omega, 0)=\left(A_{j p}+B_{j p}\right)=-\frac{h 33 \cdot I(\omega)}{i \omega A_{0}}\left[X_{2}(j p-1)+X_{2(j p-1)+1}\right](37) \\
& U\left(\omega, h_{j p}\right)=\left(A_{j p} e^{i k_{j p} h_{j p}}+B_{j p} e^{-i k_{j p} h_{j p}}\right) \\
& =-\frac{h 33 \cdot I(\omega)}{i \omega A_{0}}\left[X_{2(j p-1)} e^{i k_{j p} h_{j p}}+X_{2(j p-1)+1} e^{-i k_{j p} h_{j p}}\right]
\end{aligned}
$$

Substituting (37) and (38) into (22) and using (21) gives:

$$
\begin{aligned}
& V(\omega)=-\frac{h 33^{2} I(\omega)}{i \omega A_{0}} \cdot\left\{\left[X_{2(j p-1)}+X_{2(j p-1)+1}\right]\right. \\
& \left\{-\left[X_{2(j p-1)} e^{i k_{j p} h_{j p}}+X_{2(j p-1)+1} e^{-i k_{j p} h_{j p}}\right]\right\}-\frac{\beta 33_{j p} I(\omega) h_{j p}}{i \omega A_{0}}
\end{aligned}
$$

Dividing through by $I(\omega)$, (39) can be expressed in terms of the electrical impedance:

$$
\begin{aligned}
& Z(\omega)=-\frac{h 33^{2}}{i \omega A_{0}} \cdot\left\{\left[X_{2(j p-1)}+X_{2(j p-1)+1}\right]\right\} \\
& \left\{-\left[X_{2(j p-1)} e^{i k_{j p} h_{j p}}+X_{2(j p-1)+1} e^{-i k_{j p} h_{j p}}\right]\right\}-\frac{\beta 33_{j p} h_{j p}}{i \omega A_{0}}
\end{aligned}
$$

This result gives the complex electrical impedance as a function of frequency and contains the layered material system response function, the sensor/instrumentation response function, and the electric field pulse response. $Z(\omega)$ is then calculated from the physical parameters in (40) and using (34a) and (34b) in (35) to obtain the $X$ array. Assuming a Gaussian sensor/instrumentation response function and a square wave (time domain) electric field response for computing oq from (12), the ultrasonic waveform can be obtained by taking the real part of the inverse complex fast Fourier transform of (40).

$p(t)=\operatorname{Re}(i c f f t[Z(\omega)])$

This theory was first developed and debugged in Mathcad and then written in LabVIEW and implemented in our PEA control program.

\section{RESULTS}

Fig. 6 shows a comparison of the ray-tracing model, fullwave model and a PEA measurement for a material system containing sensor backing, PVDF sensor, $\mathrm{Al}$ electrode, 5mil Kapton dielectric film, and Al electrode (see Fig. 3). For the ray-tracing model, waveform calculations are show for 2, 6, and 10 rays which shows how the waveform from this model can progressively be made to look more and more like the measured waveform as wavelets are added.

The real PEA material system contains bonding layers which introduce closely spaced multiple reflections after many of the major peaks in the waveform. In the fullwave model, it is easy to add or take away material layers in the simulated PEA system, so these bonding layers were added to the full-wave model calculation but not to the ray-tracing model calculation. It is noted that the fullwave calculated waveform matches better with the measured waveform than with the ray-tracing model. Some of the small differences between model calculations and the measurement are most likely due to the estimated values for the attenuation coefficients and possible differences in the densities and wavespeeds from actual materials. Many of these properties needed, especially the attenuation coefficient, are not readily available, particularly for the coupling layers which may be glue or a light machine oil, for example.

As mentioned, a benefit of using a ray-tracing model is that individual wavelets (rays) can be easily included or not in the calculation. This convenience makes it easy to determine where each ray actually comes from and the route it took. This is helpful in understanding what each peak means in a measured waveform. 


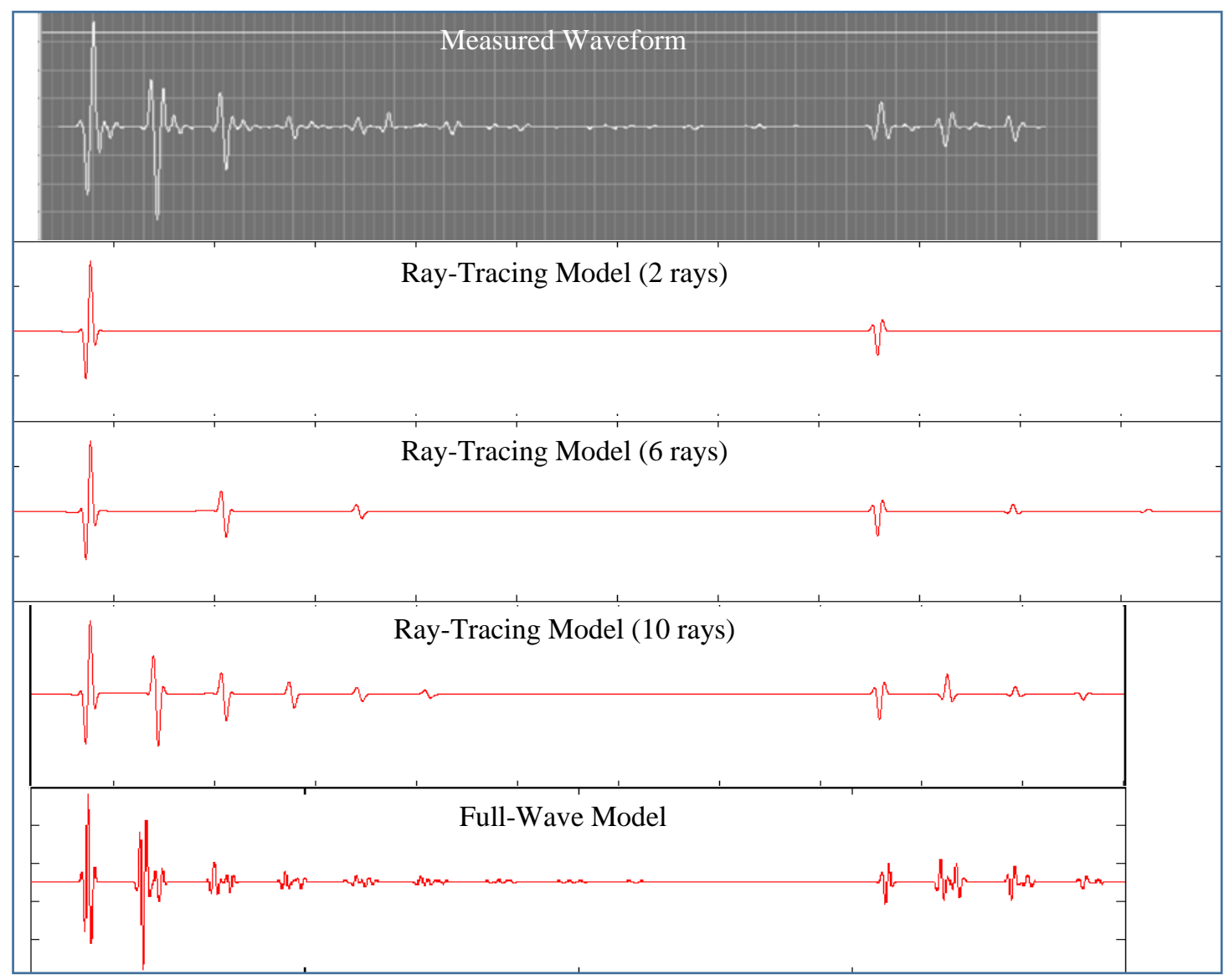

Fig. 6. Comparison of Measured Waveform and Model Calculations.

The number of layers that can be added to the full-wave model is unlimited and requires no modifications to the math that goes into the model. Adding layers to the raytracing model dramatically increases the number of rays needed to properly model a measured waveform expected from the PEA system. When ray-tracing, each wavelet must be explicitly added, mathematically, whereas, all wavelets are implicit in the full-wave model.

Because of the ease in adding layers, the full-wave model makes it easier to study the effect of coupling layers such as glues or compliant rubber layers, and also increases the ease in studying the addition of acoustic impedance matching layers. Both models can be used to study the effects caused by changing the properties of the sensor backing material.

The time scales in all of the waveform plots in Fig. 6 are the same, but the vertical scales will not be the same unless the wave amplitude is calibrated. Each model needs a different calibration factor to make the amplitude the same as the measured waveform.

\section{SUMMARY}

The models presented above compute the waveform, first in frequency domain; by then performing an inverse Fourier transform, the time-domain waveform is obtained. Analyzing computational results in both time domain and frequency domain yields the following observations:

(i) If $E(t)$ is a half-cycle square wave, which is commonly used in the PEA method, the theory predicts the generation of two waves going opposite directions from each other and out of phase with each other by $180^{\circ}$. (ii) The Fourier transform of a half-cycle square wave (as is commonly used) produces a sinc function centered at zero Hz. The piezoelectric sensor frequency response, on the other hand, is centered at its natural resonant frequency and has a response that tends to zero at zero Hz. Overlaying these two responses leads one to the conclusion that much of the energy transferred into the ultrasonic wave by the electric field impulse is filtered out by the sensor. (See the frequency domain plot in Fig. 3)

(iii) It may be better for $E(t)$ to be a one-cycle toneburst with center frequency equal to the piezoelectric sensor center frequency.

(iv) The broader the bandwidth of the sensor, the narrower the time domain response and therefore, the higher the spatial resolution. Also, the broader the bandwidth, the more the signal will look like the charge distribution without doing a deconvolution. This will happen if the sensor is essentially critically damped so that the sensor displacement follows the charge generated wave without over-shooting or ringing.

A benefit of using a ray-tracing model is that individual waves (rays) can be included or not in the calculation. 
This convenience makes it easy to determine where each ray actually comes from and the route it took. This is helpful in understanding measured waveforms.

In summary, signal processing and modeling tools have been developed, debugged, and proven out; they have been found to be beneficial in improving waveforms and analyzing their meaning and content.

\section{REFERENCES}

1. Arnaout, M., Berquez, L., Baudoin, F., and Payan, D. (2010). Contribution to Improving the Spatial Resolution of a Pulsed Electro Acoustic Cell Measurement, IEEE Int. Conf. Solid Dielectrics, Germany.

2. Dennison, J.R. \& Pearson, L.H. "Pulse Electro-Acoustic (PEA) Measurements of Embedded Charge Distributions,” (2013). Proc. SPIE Optics and Photonics Conf., 8876, 887612-1-887612-11.

3. Newhouse, V.L., et.al., (1982). Flaw-to-Grain Echo Enhancement by Split-Spectrum Processing, Ultrasonics, Mar, p. 59.

4. Honarvar, F., et.al., (2004). A New Signal Processing Technique for Enhancement of Ultrasonic Testing Signals, Proceedings of the World Conference on NDT, Montreal, Canada.

Griseri, V., et. al., (2004). Pulsed Electro-Acoustic

Technique Applied to In-situ Measurement of Charge Distribution in Electron-irradiated Polymers, IEEE Trans. Dielectrics and Electrical Insulation, 11(5), 891.

6. Laurenceau, P., Dreyfus, G., and Lewiner, J., (1977). New Principle for the Determination of Potential in Dielectrics, Phys. Rev. Lett. 38(1), 46.

$7 . \quad$ Sessler, G. M., West, J. E., Berkley, D. A., and Morgenster, G., (1977). Determination of Spatial Distribution of Charges in Thin Dielectrics, Phys. Rev. Lett. 38(7), 368.

8. Gallot-Lavallée, O., Griseri, V., Teyssedre, G., and Laurent, C., (2005). The Pulsed Electro-Acoustic Technique in Research on Dielectrics for Electrical Engineering: Today's Achievements and Perspectives for the Future, RS - RIGE. 8(5-6), 749.
Griseri, V., Bouchareb, S., Berquez, L., (2012). Adaptation of the LIMM Technique Data Treatment to Perform Measurements in Vacuum, 2012 IEEE Conference on Electrical Insulation and Dielectric Phenomena (CEIDP), Montreal, Quebec, Canada, October 14-17.

10. Miyake, H., Numata, S., Tanaka, Y., and Takada, T., (2010) Characteristic of Internal Charge Distribution in PI Irradiated by Proton, $10^{\text {th }}$ Spacecraft Charging Tech. Conf., Albuquerque, NM.

11. Griseri, V., et. al., (2006). Space-Charge Detection and Behavior Analysis in Electron-irradiated Polymers, IEEE Trans. Plasma Sci., 34(5), 2185.

12. Maeno, T., et. al., (1988). Measurement of Spatial Charge Distribution in Thick Dielectrics Using the Pulsed Electroacoustic Method, IEEE Trans. Electrical Insulation, 23(3), 433.

13. Takada, T., et.al., (1998). Comparison Between the PEA Method and the PWP Method for Space Charge Measurement in Solid Dielectrics, IEEE Trans. On Dielectrics and Electrical Ins., 5(6), 944. 14. Takada, T., Miyake, H., and Tanaka, Y., (2006). Pulse Acoustic Technology for Measurement of Charge Distribution in Dielectric Materials for Spacecraft, IEEE Transactions on Plasma Science, 34(5), 2176.

$15 . \quad$ Tashima, D., Kurosawatsu, K., Sung, Y-M., Otsubo, M., and Honda, C., (2007). Surface Modification of Nanoporous Materials for Electric Double Layer Capacitors Application, Materials Chemistry and Physics, 103(1), 158.

16. Galloy, L., Berquez, L., Baudoin, F., and Payan, D. PSpice modeling of the Acoustic Detector for a Pulsed Electro-Acoustic Cell. Proc. 2013 IEEE Conference on Solid Dielectrics, Bologna, Italy, June 30-July 4, 2013.

17. Pearson, L., "Piezoelectric Sensors for In-Situ Monitoring of Mechanical Property Changes," Proceedings of the Joint Army-NavyNASA-Air Force (JANNAF) $14^{\text {th }}$ Nondestructive Evaluation (NDES)/23 ${ }^{\text {rd }}$ Rocket Nozzle Technology (RNTS) $/ 36^{\text {th }}$ Structures \& Mechanical Behavior (S\&MBS) Joint Subcommittee Meeting, New Orleans, LA, 2004. 